\title{
Le lac Victoria (Afrique de l'Est) malade de la perche du Nil : réalité, mythe ou mystification?
}

\author{
Didier Paugy, Christian Lévêque \\ Écologues, directeurs de recherche IRD, UR131 Amazone, MNHN, CP 26, 43 rue Cuvier, 75231 Paris cedex 05, France
}

\begin{abstract}
Mots-clés :
Afrique ;

lac Victoria ;

biodiversité ; introduction d'espèces; perche du Nil est la cause de l'eutrophisation du lac. Celle-ci date de longtemps (bien avant l'introduction eutrophisation

Résumé - Le film Le Cauchemar de Darwin se présente comme un documentaire. Il s'agit en réalité d'une fiction. L'excellent accueil réservé à ce film doit beaucoup aux chocs visuels qui dénoncent les drames que subissent les populations africaines. Mais ces calamités ne forment qu'un amalgame suspect et peu crédible. L'aspect écologique est survolé et les conclusions sont fausses. Il en est ainsi de l'affirmation que la du prédateur) et elle est essentiellement liée aux activités humaines. L'approche économique est, elle aussi, caricaturale; elle ne peut se limiter à dénoncer le seul intérêt du Nord en laissant de côté les retombées de l'essor de la pêche sur le pouvoir d'achat des communautés riveraines. La malnutrition dans le secteur de Mwanza, dont parle également le documentariste, est une affabulation totale. Enfin, le fil rouge de ce film est le trafic d'armes. Étant incompétents en ce domaine, nous ne saurions attester de la véracité ou non de cette affirmation. Mais plusieurs contre-enquêtes n'ont pas réussi à prouver qu'un tel trafic couvre le transport des filets de poisson.
\end{abstract}

Keywords:
Africa;
Lake Victoria;
biodiversity;
species introduction;
eutrophication

\section{Préambule}

Le film Le Cauchemar de Darwin a fait l'objet de nombreuses polémiques. D'abord encensé par la critique, il a été par la suite fortement critiqué et l'on n'a pas hésité à parler de manipulation. Il n'a rien d'un documentaire, en effet. Hubert Sauper, son réalisateur, ne cachait pas ses intentions lorsqu'il disait : «On ne trouverait pas de

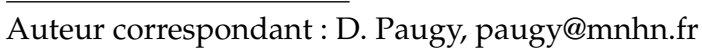

\begin{abstract}
While looking like a documentary, the film Darwin's Nightmare is, in fact, a tale and a fiction. The excellent reception reserved to this film mainly results on the shock photos, which point out the dramas undergone by the African populations. But the amalgam of all calamities (prostitution, Aids, malnutrition, war, etc.) attributed to Nile perch introduction is not credible. The ecological side is quite weak and provides false conclusions. For instance, it is not true to pretend that the introduction of the Nile Perch is the main cause of the lake's eutrophication. This phenomenon lasted since a long time (many years before the introduction of the predator) and is a result of human activities. The economic side is also very caricatural and it cannot obviously be resumed to the denunciation of the North exploiting the South. It wilfully ignores the consequences of the improved fishery on the purchasing power of the bordering human communities of the lake. The film also point out the malnutrition in the Mwanza district. That is a not an accepted fact around the lake. Lastly, the red wire of this film is the traffic of weapons. As we are unskilled in this field, it is impossible for us to check the reality of this traffic. But several counter-enquiries failed to prove that such traffic supports the transport of the fish fillets.
\end{abstract}

\footnotetext{
${ }^{1}$ Propos cités (p. 8-9) dans le dossier préparé par Françoise et Jean-Paul Eckstein, à l'occasion des Rencontres cinématographiques d'Alsace de novembre 2005, où le film fut présenté.
}

perche du Nil dans nos supermarchés s'il n'y avait pas de guerre en Afrique ${ }^{1}$.»

Pourquoi et comment est née la polémique autour de ce film? Ce sont des questions auxquelles nous essayons d'apporter quelques éléments de réponse, en cherchant à comprendre comment une opinion très favorable au départ a pu évoluer en moins d'un an (Encadré 1).

Article published by EDP Sciences and available at http://www.nss-journal.org or http://dx.doi.org/10.1051/nss:2008005 


\begin{abstract}
Encadré 1. Discussions et controverses
Elles peuvent être résumées à partir d'articles repérés dans des journaux ou revues français. Le débat s'est déroulé selon quatre grandes étapes chronologiques : les louanges d'un documentaire-culte, la dénonciation d'un trafic d'armes et la bannière des altermondialistes (Libération: Sylvie Briet et Laure Noualhat, 2 mars 2005; Télérama : Pierre Murat, 5 mars 2005 ; Le Point : François-Guillaume Lorrain, 31 mars 2005...), puis une difficile riposte des scientifiques (Le Monde : Didier Paugy, 10 mars 2005 ; Politique africaine, 98 : Christine Deslaurier, juin 2005 ; Eaux libres, 41 : Christian Lévêque et Didier Paugy, juillet 2005 ; Métro : Christian Lévêque, 8 novembre 2005), enfin, le débat s'instaure (Les Temps modernes, 635-636 : François Garçon, janvier 2006)... suivi de la règle des « $3 \mathrm{~L} »^{2}$ (Télérama : Thierry Leclère, $1^{\mathrm{er}}$ mars 2006 ; Le Monde : Jean-Philippe Rémy, 4 mars, 2006 ; Charlie Hebdo : Antonio Fischetti, 15 mars 2006 ; Libération : Sylvie Briet, 26 novembre, 2006).

Et bien d'autres dont on trouvera une liste, sinon exhaustive, du moins très complète dans le livre fort bien documenté de F. Garçon (2006b).
\end{abstract}

Dans l'introduction de son excellent ouvrage, L'Afrique des idées reçues, Georges Courade (2006) écrit : «Le Cauchemar de Darwin, le film d'Hubert Sauper, donne une excellente synthèse de cette Afrique victime. » Suivent quelques lignes où l'auteur résume les images laissées par H. Sauper, pour conclure : "Alors que la Tanzanie est touchée par la famine, les perches s'envolent vers l'Europe et ne restent que les carcasses de poissons pour la consommation locale [...]. »

Comment un africaniste de la trempe de G. Courade a-t-il donc pu se laisser abuser par ce film ? Comment tant de spectateurs ont-ils fait de ce soi-disant documentaire ${ }^{3}$ un film-culte? Et comment autant de critiques élogieuses ont-elles pu être unanimement diffusées, quelle que soit la couleur politique du média? Comme le relève F. Garçon (2006a), «à cette occasion un cecuménisme rarissime [s'est établi] pour un film qui tient un discours ouvertement militant d'inspiration antimondialiste ».

En réalité, personne ne semble s'être posé la question de la "véracité de ce qui est annoncé, de l'authenticité, de l'éthique du réalisateur ». Il était acquis qu'il s'agissait d'un film-vérité, puisque $H$. Sauper a toujours précisé que son film se contentait de témoigner. Un témoignage à charge pour étayer sa thèse selon laquelle la paupérisation de l'Afrique est en grande partie sous influence du marché mondial (cf. Almarcha, 2005). Mais, à notre avis, un faux témoignage qui n'hésite pas à manipuler les images pour toucher la corde sensible du spectateur et culpabiliser les Occidentaux en mal de bonne conscience. Ceux, par exemple, qui pratiquent l'autoflagellation, un phénomène qui fut dénoncé, il y a plus de vingt ans, sous le nom de «sanglot de l'homme blanc ${ }^{4}$ ».

Selon les contre-enquêtes de journalistes, trois aspects du film posent problème : l'éthique qu'il véhicule; son analyse des retombées économiques liées à la perche; ses

\footnotetext{
${ }^{2}$ La règle des « $3 \mathrm{~L}$ » a été énoncée par Jean-François Khan : «Je Lèche, je Lâche, je Lynche. »

${ }_{3}$ Ce film a reçu, entre autres récompenses, le prix du meilleur documentaire (Montréal, 2004) et a été sélectionné en 2006 pour l'Oscar du meilleur documentaire (qui sera finalement décerné à La Marche de l'empereur).

4 Pascal Bruckner, 1983. Le Sanglot de l'homme blanc, Paris, Éditions du Seuil.
}

\section{Encadré 2. Une supercherie démasquée}

Si elle fut longue à se dessiner, la supercherie que contient ce film a, néanmoins, fini par être dénoncée. Nous l'avions proclamée très tôt $t^{5}$, mais tout commence véritablement après qu'un journaliste du Monde, interpellé par le brûlot de F. Garçon (2006a), soit allé enquêter à Mwanza (en Tanzanie), lieu du tournage. Dans son article "Contre-enquête sur un cauchemar ", Jean-Philippe Rémy (Le Monde, 4 mars 2006) dresse un réquisitoire sans appel et affirme que Mwanza profite très largement du succès du poisson. D'ailleurs, à propos de l'idée que les pays riches puissent cesser d'acheter de la perche, l'avocat James Njelwa (défenseur des pêcheurs contre les industriels de la pêche) n'hésite pas à se prononcer : «Mais ils sont fous! Ils veulent nous condamner à la pauvreté? » C'est une réponse catégorique à ceux qui ont appelé à un boycott de la perche sans s'interroger sur le bien-fondé et les conséquences d'une telle mesure - si ce n'est pour se donner bonne conscience. Certainement vexés de s'être ainsi "fait berner ", d'autres journalistes ont également mené une contreenquête, pour arriver à la même conclusion ou du moins mitiger leurs précédents éloges ${ }^{6}$. Encore la règle des « $3 \mathrm{~L} » .$.

affirmations sur le trafic d'armes. Rien ou presque, en revanche, n'a été écrit concernant l'écologie et l'environnement du lac Victoria. C'est donc notre rôle de biologistes et d'écologues de dénoncer les erreurs ou les contrevérités qui affleurent dans le film. Nous ne nous prononcerons pas ici sur le prétendu trafic d'armes permettant la rentabilisation des voyages allers. Sur ce point, F. Garçon (2006b) a suffisamment enquêté pour que nous puissions nous faire une opinion quant aux méthodes employées par H. Sauper pour asseoir son scénario (Encadré 2).

\footnotetext{
${ }^{5}$ Cf. Encadré 1.

6 Sylvie Briet et Laure Noualhat, Libération, $1^{\mathrm{er}}$ mars 2006 (l'article rapporte l' « échange de vues musclé » entre F. Garçon et $\mathrm{H}$. Sauper qui a eu lieu dans les locaux du quotidien); Michel Guérin et Jacques Mandelbaum, Le Monde, 28 mars 2006 ; Antonio Fischetti, Charlie Hebdo, 17 mai 2006; Anne Crignon, Le Nouvel Observateur, $1^{\text {er }}$ juin 2006.
} 


\section{Les grands lacs de l'Afrique de l'Est: "viviers de Darwin 7 ", véritables laboratoires pour l'étude de l'évolution}

Au Tertiaire, un gigantesque phénomène de fracturation, qui se poursuit de nos jours, affecte la partie orientale du continent africain. L'Afrique de l'Est se détache progressivement du reste du continent. Des fossés d'effondrement se créent et forment le rift est-africain, un creuset de vie (voir «Lucie», par exemple) dans lequel se sont formés les grands lacs d'Afrique de l'Est (Fig. 1A).

Ce sont les lacs de la démesure, tant en ce qui concerne leur volume (lac Tanganyika : $18900 \mathrm{~km}^{3}$ ), leur profondeur (lac Tanganyika : $1470 \mathrm{~m}$ ), leur surface (lac Victoria : $68800 \mathrm{~km}^{2}$ ), que la richesse de leur faune (lac Malawi : plus de 800 espèces de poissons dont près de 800 Cichlidés). La faune des lacs d'Afrique de l'Est est à la fois très riche et très diversifiée, avec des centaines d'espèces de Cichlidés endémiques de chacun de ces lacs.

Pour les biologistes, les lacs de l'Est de l'Afrique sont ainsi de véritables laboratoires où l'on peut observer de spectaculaires radiations adaptatives uniques au monde. La plasticité morphologique et biologique des poissons Cichlidés a engendré une incroyable diversité de formes et de comportements, au point que l'on estime actuellement qu'il existe, uniquement dans cette zone, environ 2500 espèces.

La spéciation particulièrement rapide des ces «essaims d'espèces » a pu se faire par l'utilisation de nombreuses niches écologiques et par l'adaptation des stratégies biologiques et écologiques. On ne retrouve aucune espèce commune dans chacun des lacs, alors que beaucoup se ressemblent étrangement. Ces convergences signifient que des adaptations similaires sont apparues plusieurs fois de manière indépendante.

Sur la base de la distribution des espèces, on distingue un certain nombre de communautés ichtyologiques qui occupent les différentes zones des lacs. Dans chaque communauté, il existe des espèces étroitement confinées à leur habitat spécifique et d'autres qui possèdent une plasticité beaucoup plus grande et sont susceptibles de se déplacer d'un milieu à l'autre. Enfin, des adaptations particulières liées aux stratégies et aux comportements de reproduction ont largement favorisé l'existence de barrières entre espèces, suscitant ainsi spéciation et diversité.

Le lac Victoria s'est formé depuis environ 500000 ans. Jusque récemment, on pensait qu'il s'était asséché il y a environ 14000 ans et que la faune actuelle de poissons

\footnotetext{
${ }^{7}$ L'expression fait référence au Vivier de Darwin, ouvrage de Tijs Goldschmidt (2003), dont un compte rendu par M.-A. Hermitte a été publié dans Natures Sciences Sociétés, 13, 2 (2005), 216-218.
}

ne pouvait s'y être établie que depuis. Or, les marqueurs génétiques montrent que la faune de Cichlidés du lac s'est différenciée il y a environ 200000 ans. Donc, le lac ne s'est certainement pas asséché totalement, et l'hypothèse la plus plausible (celle qui prévaut actuellement) est que des zones marécageuses auraient subsisté. Celles-ci auraient alors fourni un refuge aux poissons.

Un consensus semble être désormais établi quant à l'origine de la faune ichtyologique du lac Victoria (Nishida, 1991 ; Stiassny et Meyer, 1999; Meyer, 2005 ; Salzburger et al., 2005). Des lignées ancestrales de Cichlidés venant de l'ouest (région congolaise ou camerounaise?) ont tout d'abord colonisé le lac Tanganyika, qui est le plus ancien. Ainsi, le lac Tanganyika serait le «berceau » des Cichlidés Haplochromines de la région. La diversité génétique des espèces montre qu'elles dérivent de onze ou douze espèces ancestrales (Stiassny et Meyer, op. cit.). De ce lac, plusieurs lignées ont ensuite émigré pour coloniser une grande partie de la sous-région. C'est ce que l'on a coutume d'appeler le « out of Tanganyika ». C'est ainsi que les premiers Haplochromines sont apparus dans les lacs Victoria et Malawi. Dans le cas du lac Victoria, cependant, des Haplochromines seraient également originaires de certains lacs voisins, comme les lacs Albert, Édouard, Georges, Kyoga et Kivu (Salzburger et Meyer, op. cit.).

\section{L'origine des introductions : une nécessité, pas un hasard, encore moins une expérimentation!}

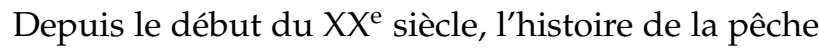
dans le lac Victoria a été marquée par la surexploitation progressive des stocks de poissons. Dès cette époque, certains proposaient d'ailleurs de limiter la période d'exploitation. Néanmoins, avant toute mesure restrictive, on jugea préférable d'étudier auparavant la biologie des espèces, et c'est ainsi que fut créé l'East African Fisheries Research Organisation (EAFRO), à Jinja, sous la direction de Michael Graham et Edgar B. Worthington.

Dans les années 1950-1960, l'utilisation systématique d'engins très destructeurs, comme le chalut de fond, a rapidement entraîné un épuisement des stocks, notamment en Tanzanie. Il est, dès lors, apparu évident que les stocks de plusieurs espèces locales, d'intérêt commercial, avaient pratiquement disparu (Ogutu-Ohwayo, 1990 ; Witte et al., 1992). Face à ce déclin des espèces prisées des populations riveraines, l'exploitation se reporta, en partie, à la fin des années 1960 sur les Haplochromines, peu appréciés jusqu'alors (ils avaient d'ailleurs reçu le nom significatif de «bony fishes »).

C'est, entre autres, pour pallier cette baisse des captures qu'il avait été proposé, au début des années 1950, 


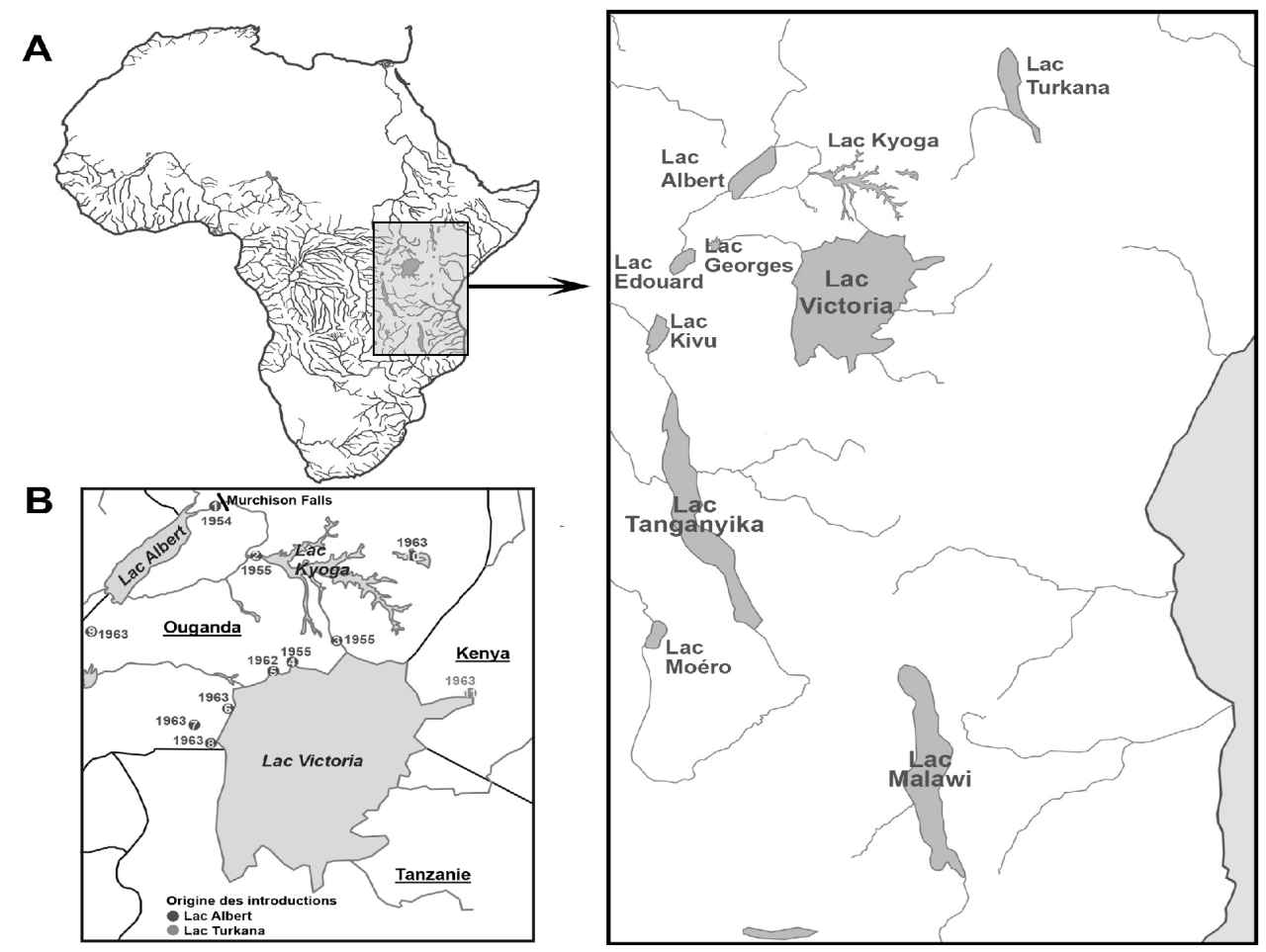

Fig. 1. A : Afrique de l'Est et lacs de la vallée du Rift. B : localités et années où furent pratiqués les différents lâchers de Lates niloticus (d'après Pringle, 2005).

d'introduire quatre espèces allochtones de «tilapias ${ }^{8}$ ». Un débat opposa rapidement les scientifiques aux autorités coloniales, qui pensaient que la production halieutique serait nettement accrue si des espèces allochtones, judicieusement choisies, étaient introduites, puisque les Haplochromines ne représentaient qu'une piètre ressource et une médiocre nourriture. Ils n'étaient pas rares ceux qui pensaient alors que des espèces natives du lac Albert, proche (Fig. 1A), pourraient être implantées dans le lac Victoria. En 1953, sans que l'on en connaisse la raison exacte, mais certainement parce que les scientifiques ne s'étaient pas montrés assez persuasifs, l'EAFRO finit par se laisser convaincre et autorisa l'introduction controversée de quatre espèces de tilapias non endémiques (Oreochromis leucosticus, O. niloticus, Tilapia rendalli et T. zillii).

Mais, face à l'introduction de grands prédateurs, en l'occurrence la perche du Nil (Lates niloticus), l'EAFRO s'est toujours montré ferme, car les scientifiques estimaient que l'essor naissant des importantes pêcheries de tilapias pouvait être ruiné. Notons, au passage, que le sort des Haplochromines n'est jamais évoqué. Néanmoins, il ne s'agissait pas d'un refus définitif; les scientifiques

\footnotetext{
8 Le terme générique « tilapia » est employé à dessein, car il permet de regrouper plusieurs espèces appartenant ou ayant appartenu à ce genre désormais éclaté en plusieurs autres. Le mot «tilapia » est d'ailleurs passé dans le langage courant des Africains francophones (les anglophones ont l'équivalent «tilapines »).
}

voulaient simplement se laisser le temps d'étudier l'impact de l'introduction d'un prédateur sur les espèces en place. À titre expérimental, on proposa d'introduire des Lates dans le lac Nabugabo, petit lac satellite du lac Victoria. Si un équilibre entre les populations du prédateur et celles des espèces présentes dans le lac se maintenait, on pourrait alors envisager l'introduction de la perche dans le lac Victoria proprement dit. Ce principe de précaution n'était pas nouveau et ce type d'expérimentation avait d'ailleurs déjà été énoncé plusieurs années auparavant (Graham, 1929; Worthington, 1932). Quoi qu'il en soit, ces études sont nécessairement longues et les autorités pressèrent les scientifiques de donner rapidement des résultats convaincants. On se trouvait, en effet, en pleine période de récession, notamment alimentaire, suite aux ravages de la Seconde Guerre mondiale. Il faut avouer que, dans ce contexte, le seul argument "préservation de la biodiversité » n'eut que peu de poids contre les pressions politiques. Face aux arguments économiques avancés, les scientifiques se montrèrent incapables de trouver une quelconque valeur commerciale aux «trash Haplochromines ». Pour sauver la face, on argumenta alors que l'étude de l'impact de l'introduction du Lates dans le lac Nabugabo n'était pas achevée. L'Uganda Game and Fisheries Department (UGFD) ${ }^{9}$, qui prônait l'introduction de la perche du Nil, rétorqua que des fossiles cousins

\footnotetext{
9 L'UGFD peut être considéré comme l'équivalent ougandais de notre Office national des forêts.
} 
du Lates avaient existé dans le lac et avaient disparu. Il convenait donc de les réintroduire!!! L'UGFD s'appuya enfin sur un vague rapport mentionnant que des perches avaient été aperçues dans le lac près de Port Bell (Pringle, 2005).

En fin de compte, face à des agents de l'UGFD déterminés, les scientifiques ne réussirent pars à convaincre les décideurs de la dangerosité de l'introduction d'un grand prédateur. Les lâchers de Lates niloticus furent effectués en deux grandes vagues (Pringle, op. cit.). En 1954, quelques spécimens, provenant du lac Albert, furent introduits aux environs des Murchison Falls dans le Nil Victoria (Fig. 1B). Cette introduction initiale, comme les suivantes, est de la responsabilité de l'UGFD et non de l'initiative d'un personnage muni d'un seau contenant des perches, comme le prétend H. Sauper. Ce premier essai permit de conclure que la perche du Nil s'acclimatait convenablement. Face à ce succès, d'autres introductions furent alors pratiquées en Ouganda. Six années plus tard, les premiers spécimens étaient signalés à Mwanza, en Tanzanie, donc dans le sud du lac. Une seconde campagne eut lieu en 1963, avec quatre nouveaux lâchers ${ }^{10}$ : trois en Ouganda et un au Kenya (Fig. 1B).

\section{Qui est la «perche du Nil »?}

La perche du Nil (Lates niloticus), traduction littérale de l'anglais Nile perch, encore appelée « capitaine ${ }^{11}$ » en Afrique de l'Ouest, appartient à la famille des Latidae (Nelson, 2006). Le genre Lates comprend huit espèces, dont sept vivent en eau douce et cinq en Afrique ${ }^{12}$. Parmi les cinq espèces africaines, quatre sont endémiques du lac Tanganyika (dont Lates angustifrons, qui peut atteindre $80 \mathrm{~kg}$ ), où elles cohabitent en parfaite harmonie avec des Cichlidés, eux aussi endémiques.

Naturellement, Lates niloticus existe dans tous les bassins de la zone nilo-soudanienne, y compris dans les lacs Albert et Turkana (Fig. 2), proches du lac Victoria et d'où proviennent les spécimens introduits. C'est le plus gros poisson d'eau douce d'Afrique. Les plus gros spécimens peuvent mesurer plus de 1,50 m et peser plus de 100 kg. Il s'agit là de valeurs extrêmes, mais des poissons avoisinant $50 \mathrm{~kg}$ sont assez fréquents. C'est aussi un carnivore vorace qui avale entières, grâce à sa très large gueule, toutes les proies qui passent à sa portée.

\footnotetext{
10 Nous ne parlons ici que des introductions effectuées dans le lac Victoria proprement dit. D'autres lâchers, à cette occasion, ont été opérés dans plusieurs autres lacs satellites.

11 À ne pas confondre avec l'espèce marine Polydactylus quadrifilis (Perciformes, Polynemidae), appelée elle aussi «capitaine», notamment au Sénégal et au Togo.

12 On a longtemps considéré qu'il existait sept espèces en Afrique, mais les deux formes des lacs Albert et Turkana appartiennent bien à l'espèce L. niloticus (Agnèse, communication personnelle).
}

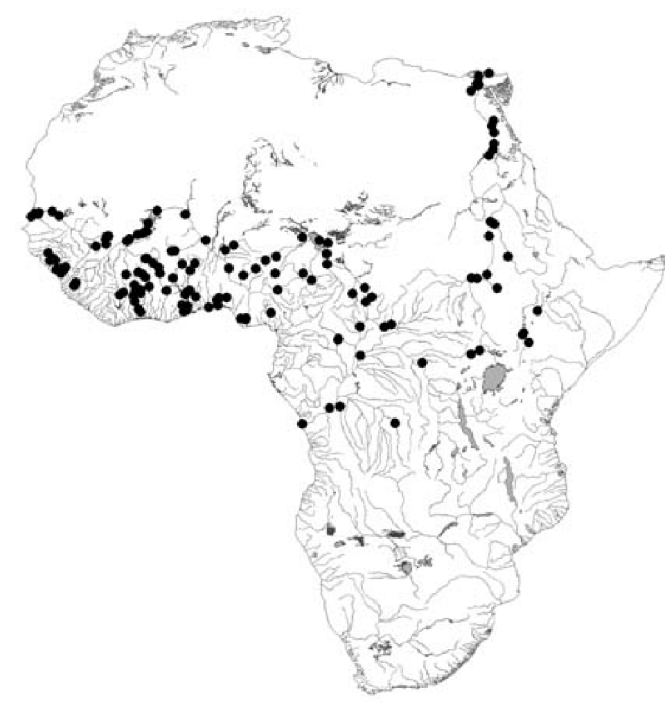

Fig. 2. Lates niloticus : répartition naturelle de l'espèce.

Poisson recherché par les pêcheurs sportifs, il représente pour les pêcheurs locaux une providence économique très intéressante. En revanche, les populations locales s'en nourrissent peu - on connaît, à cet égard, la réticence habituelle des populations à consommer une nourriture nouvelle. De plus, n'ayant pas la pratique du fumage ou du séchage de spécimens de grosse taille, elles n'ont pas trouvé initialement la façon de le conserver.

\section{La perche du Nil, cause unique de la destruction de la biodiversité ?}

Après l'introduction du Lates, beaucoup d'avis concordèrent pour affirmer que les Haplochromines étaient menacés de disparition. Dans la mesure où il y a eu un certain synchronisme entre la pullulation du Lates et la disparition des Haplochromines, on accusa bien évidemment le Lates. Nous avons nous-mêmes évoqué un «effet Frankenstein » vis-à-vis de proies un peu naïves qui se sont retrouvées face à un superprédateur sans avoir le temps de développer des comportements d'évitement, comme cela a pu être le cas dans le lac Tanganyika.

D'autres espèces avaient disparu bien avant son introduction. Mais il s'agissait d'espèces largement exploitées par les pêcheurs et consommées par les populations, ce qui n'était pas véritablement le cas des Haplochromines. Existe-t-il d'autres hypothèses, en dehors de la prédation par le Lates, qui permettent d'expliquer la raréfaction des Haplochromines? La réponse est oui, mais elles ont été négligées ou minimisées devant l'évidente, et bien commode, «culpabilité » de la perche du Nil. Celle-ci a sans aucun doute joué un rôle, mais elle n'est certainement 
$\mathrm{qu}^{\prime}$ un facteur parmi d'autres, comme :

- l'utilisation d'engins de pêche de plus en plus performants ;

- l'eutrophisation du lac, qui a débuté dès les années 1930. En profondeur, la couche anoxique est remontée d'environ 20 m (Lévêque et Paugy, 2006);

- les mauvaises conditions écologiques générales (forte augmentation de la turbidité, par exemple), dont l'effet est d'autant plus drastique que les Haplochromines utilisent des signaux visuels de reconnaissance sexuelle;

- l'impact provoqué par l'introduction des tilapias, notamment en termes de compétition, d'alimentation et d'espace ;

- l'impact de la baisse du niveau du lac (Encadré 3).

\section{L'érosion de la diversité des Haplochromines a-t-elle une conséquence sur la pêche?}

À côté de l'aspect « conservation des espèces et du patrimoine vivant », on doit se poser la questions suivante: La destruction de la richesse en poissons a-t-elle un impact sur la biomasse globale et sur la pêche?

Une première série d'interrogations concerne la relation entre la diversité en espèces et la stabilité des écosystèmes. Le débat est toujours ouvert parmi les écologistes pour savoir si les systèmes les plus complexes, du point de vue de la composition spécifique et des réseaux d'interactions, sont également les systèmes les plus stables vis-à-vis des perturbations externes. L'expérience de l'introduction des Lates dans le lac Victoria tend à montrer que la diversité spécifique et la grande variété des spécialisations trophiques qui existaient dans ce lac n'ont pas joué en faveur d'une résilience du système écologique, qui a été fortement modifié et simplifié. Mais, simultanément, les écologistes reconnaissent que les introductions sont largement facilitées dans des systèmes dégradés. Est-ce le cas pour le lac Victoria, en cours d'eutrophisation?

Une deuxième série $d$ 'interrogations porte sur la relation entre la richesse en espèces et la production biologique. Les théories écologiques, concernant les relations entre la productivité biologique (et donc la production halieutique) et la biodiversité, sont rares et les points de vue sont loin d'être concordants. Un travail, sous presse, reprenant de nombreuses données recueillies au cours des dernières décennies montre qu'il n'existe pas de corrélation entre richesse en espèces et production biologique dans les systèmes aquatiques continentaux. On trouve tous les cas de figures; ce n'est donc pas une «loi » écologique.

On peut penser que la production piscicole des lacs ayant des chaînes trophiques courtes est plus importante que celle des systèmes ayant de longues chaînes trophiques, pour lesquels il existe une déperdition importante d'énergie à chaque changement de niveau trophique. Cette hypothèse est en faveur d'une relation inverse entre richesse spécifique et productivité ichtyologique, comme on le vérifie empiriquement dans les milieux d'élevage.

Dans le cas du lac Victoria, la grande diversification des petits Cichlidés s'est faite sur la base d'une utilisation d'un maximum de niches écologiques et de régimes trophiques, dans un type de fonctionnement donné de l'écosystème. Si ce dernier se modifie, cette construction se révèle fragile pour les Cichlidés endémiques, mais d'autres espèces peuvent prendre le relais, comme les tilapias, les crevettes (Caridina africana) ou les dagaas (Rastrineobola argentea).

\section{Les origines des pollutions, de l'eutrophisation et leurs conséquences}

Dans son film, H. Sauper est catégorique : « l'eutrophisation du lac Victoria est due à la prolifération de Lates niloticus ». Il aurait dû prendre conseil auprès de ses garants scientifiques.

L'eutrophisation résulte, en effet, de l'augmentation de la concentration en phosphore, azote et autres nutriments nécessaires au développement des plantes aquatiques. L'eutrophisation du lac Victoria doit être, sans nul doute, attribuée à la croissance démographique et au développement de l'agriculture le long des rives du lac depuis les années 1930 (Verschuren et al., 2002). Il n'existe pas, à proprement parler, d'estimation quantitative, actuelle et historique, des apports totaux de nutriments dans le lac. Mais on connaît, en revanche, la corrélation significative entre l'effectif de la population et la production agricole lors de ces quarante dernières années. L'historique de l'accroissement de la population peut donc servir d'indicateur pour comprendre l'impact passé et actuel qui se manifeste sur l'utilisation des sols et, donc, les conséquences du rejet de nutriments qui en résulte (Caraco, 1995). Il s'agit d'un phénomène combiné de déforestation, de labour, puis de lessivage des sols, auxquels se mêlent engrais, pesticides et rejets industriels vers l'exutoire que constitue le lac. Rappelons ici que, si la population ne s'est guère accrue autour du lac Victoria de 1900 à 1930, elle est passée de 4,6 millions en 1932 à 29,7 millions en 1995 (Scheren et al., 2000). Il semble, à cet égard, que l'achèvement de la voie ferrée entre Dar-es-Salam et Mwanza, en 1930, ait stimulé l'économie agricole en facilitant l'exportation de la production. On doit également y ajouter les progrès dans le domaine sanitaire, valables, par ailleurs, pour tout le continent. Actuellement, on estime que le bassin du lac Victoria, dans son ensemble, est certainement l'une des parties d'Afrique la plus peuplée (ibid.).

Nous ne reviendrons pas sur ce phénomène d'eutrophisation, qui a déjà fait l'objet d'une synthèse (Lévêque et Paugy, 2006). Dans les pays développés, il est possible 


\section{Encadré 3. Le lac Victoria risque-t-il de s'assécher?}

Telle est la question alarmiste qui a récemment été relayée par les médias. Elle fait suite à un rapport commandé par une ONG (International River Networks), dans lequel un expert, David Kull, impute une bonne part (55\%) de l'assèchement constaté du lac Victoria aux deux barrages situés en aval. Selon lui, pour alimenter deux centrales électriques, l'Ouganda procéderait secrètement à des lâchers d'eau supérieurs aux normes initialement prévues, qui étaient censées plus ou moins simuler l'écoulement naturel. Certes, on connaît le rôle que peuvent jouer les barrages sur le bilan hydrique des hydrosystèmes, mais il semble qu'il ne s'agisse pas là de la principale cause de la baisse de niveau du lac. On estime, en effet, que l'essentiel des apports en eau du lac proviennent des pluies (Tate et al., 2004). En tenant compte de ces apports pluviométriques et des pertes, effluents et évaporation, une modélisation climatique globale a permis de montrer que le niveau du lac continuerait vraisemblablement de baisser jusque vers 2030, puis qu'une remontée devrait s'ensuivre jusque vers les années 2080. Cette modélisation s'appuie sur un bilan hydrologique constaté sur la période 1925-2000. Cette chronique montre d'ailleurs qu'entre 1930 et 1960, le niveau réel, et non plus modélisé, du lac était bien plus bas qu'actuellement ( $c f$. tableau ci-dessous). Rappelons que les barrages incriminés ont été achevés en 1954 (Nalubaale dam, ex-Owen Falls dam) et en 2002 (Kiira dam).

On ne peut donc exclure une influence des barrages, mais il est également clair que les variations climatiques et le réchauffement global ont certainement, dans cet exemple, un impact prépondérant. Signalons incidemment que les mêmes arguments sur le rôle de l'irrigation et des barrages ont été utilisés pour expliquer l'assèchement partiel du lac Tchad. Dans ce dernier cas, il est clair pourtant que ce sont les variations de la pluviométrie (la sécheresse du Sahel) qui sont responsables de la quasi-disparition du lac, dont le niveau a lui aussi fluctué au cours du $X X^{\mathrm{e}}$ siècle!

Variation du niveau du lac Victoria entre 1930 et 2000 (d'après Tate et al., 2005).
\begin{tabular}{cc} 
Période & Niveau du lac (m) \\
\hline $1930-1960$ & $10,4-11,4$ \\
$1960-1980$ & $12,0-13,2$ \\
$1980-2000$ & $11,5-12,6$
\end{tabular}

de juguler l'eutrophisation. Cela a été fait en France, par exemple, pour les lacs alpins (Annecy, Bourget, Léman). Mais ce sont là autant de travaux très coûteux que les riverains du lac Victoria n'ont pas les moyens de s'offrir.

\section{La perche, les tilapias, les dagaas, les crevettes... et l'économie de la pêche}

Entre autres idées, H. Sauper veut faire passer dans son film celle selon laquelle le système en cours appauvrirait les populations locales, en les dépossédant des produits de la pêche exportés vers les pays du Nord.

Le premier constat est l'important accroissement de l'effort de pêche, qui s'exprime tant en ce qui concerne l'augmentation du nombre de pêcheurs que celle du nombre d'embarcations (Goudswaard et al., 2002). Ainsi, entre 1990 et 2000, le nombre de pêcheurs a plus que doublé en Tanzanie et en Ouganda. Cette tendance semble s'être encore accélérée entre 2000 et 2002 (Matsuishi et al., 2006). Cet essor se traduit bien évidemment par des chiffres : la production annuelle de poisson était inférieure à 100000 tonnes jusqu'au début des années 1980. Elle est aujourd'hui supérieure à 500000 tonnes (Fig. 3). Certes, les Lates représentent plus de la moitié des captures, et la moitié de cette pêche, convertie en filets, est exportée vers les pays du Nord. En termes de protéines, et si l'on raisonne de façon simpliste, cela est choquant, d'autant que $\mathrm{H}$. Sauper affirme également que les Tanzaniens riverains du lac souffrent de malnutrition ${ }^{13}$. À cet égard, écoutons également le père José Vincente Sotillo, qui vit depuis trente ans en Afrique et depuis douze années à Mwanza : «En douze ans, j'ai connu deux cas d'enfants atteints de sous-nutrition pathologique dans ma paroisse de Mwanza. Rien de comparable avec ce que j'ai pu voir au Congo ou au Mozambique où les enfants atteints se comptaient par centaines » (entretien cité par F. Garçon, 2006b). Encore un amalgame dont H. Sauper est décidemment friand... L'exportation représente une manne non négligeable, puisqu'elle est estimée entre 38 millions (Tanzanie) et 49 millions (Kenya) de dollars américains. Les bénéfices de la perche reviennent bien, en partie, à la population locale, puisque le pêcheur tanzanien vend le kilo de perche 0,7 US\$ à un intermédiaire qui le cède 1,5 US\$ à l'usine qui en fait des filets. Après traitement, le poisson, en filets, est vendu à 3,5 US\$ à un exportateur. Au passage, l'État tanzanien prélève $6 \%$ de taxes sur chaque kilo exporté (Modesta, 2005), ce qui est faible. Il est assez difficile de connaître le montant exact des exportations, qui ne doivent pas cependant dépasser plus de $30 \%$ du montant total de la pêche.

En ce qui concerne les protéines consommées par les riverains, n'oublions pas le tonnage important de poissons qui est consommé localement, puisque les dagaas (Rastrineobola argentea), les tilapias et, depuis peu, les crevettes (Caridina africana) représentent la moitié des captures totales effectuées par les pêcheurs (FAO, 2006).

\footnotetext{
13 Affirmation totalement fausse dont, d'ailleurs, personne n'est dupe (Anne Crignon, Le Nouvel Observateur, 10 avril 2006).
} 
Fig. 3. Évolution des captures moyennes annuelles au cours des 25 dernières années (d'après Matsuishi et al., 2006).

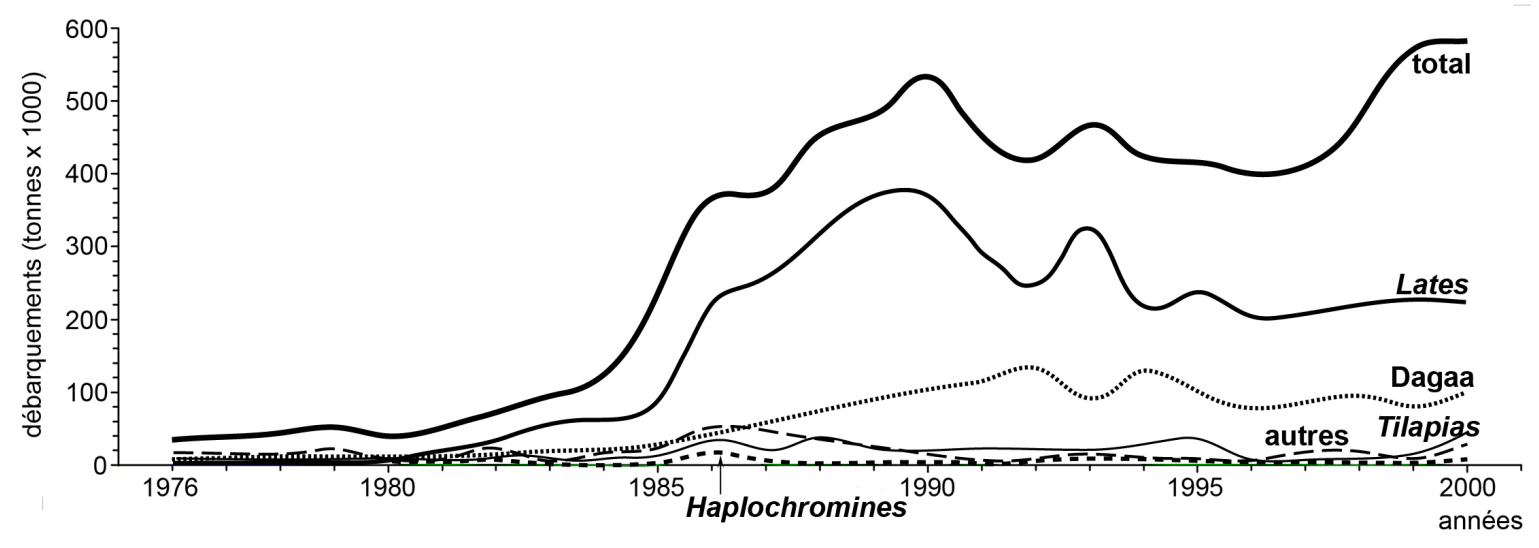

Donc, affirmer que la transformation puis l'exportation de la perche privent les populations locales de poissons mériterait d'être vérifié.

\section{Les cautions scientifiques de H. Sauper}

Pour étayer son discours, le réalisateur ne prend en compte que des articles, la plupart anciens, où sont dénoncés les « ravages » occasionnés par la prédation des Lates sur la faune des poissons endémiques. Ces articles, très nombreux dans les années 1990, dénoncent, pour la plupart, la catastrophe écologique qui menace (Kaufmann, 1992 ; Witte et al., 1992 ; Pitcher et Hart, 1995 ; Goudswaard et Witte, 1997...). Alors que des millénaires ont été nécessaires pour créer une telle diversité, il n'aurait fallu que quelques dizaines d'années au prédateur pour anéantir cette richesse. Parmi ces scientifiques, un certain nombre sont revenus, cependant, sur leurs affirmations initiales. La pullulation du Lates dans les années 1980 a coïncidé avec la disparition de poissons dont les stocks, comme nous l'avons déjà signalé, avaient déjà été mis à mal par d'autres facteurs.

Ce qui est curieux, c'est que H. Sauper n'ait pas jugé utile de s'entourer des meilleures garanties scientifiques. L. Kaufmann a une solide réputation concernant le lac Victoria, mais la lecture de la contre-enquête de F. Garçon ${ }^{14}$ (2006b) révèle une certaine manipulation. Il semble, selon lui, que, dans le dossier de presse, ne figure qu'une traduction réduite, parfois approximative, du texte original de l'Américain. Même si L. Kaufmann apporte globalement son appui au film, la version française, ainsi amputée, cache l'affirmation selon laquelle l'industrialisation de la pêche a largement profité, même inégalement, aux riverains du lac. Le scientifique écrit même : «Ce dont je peux témoigner, c'est que les projets initiaux de l'Union européenne et de la Banque mondiale, à propos du lac

\footnotetext{
${ }^{14}$ F. Garçon est historien, maître de conférences (HDR) à la
} Sorbonne, où il enseigne l'histoire du cinéma.
Victoria, sont plutôt bons » (cité par Garçon, 2006b). Néanmoins, comme solution aux besoins alimentaires du pays, L. Kaufmann reste un fervent défenseur d'une intervention publique massive, que la Tanzanie a déjà subie sous J. Nyerere, et stigmatise l'intervention privée. Le Kenya et l'Ouganda voisins auraient certainement pu servir de comparaison.

Eirik G. Jansen est la deuxième caution scientifique de H. Sauper. Pour cet économiste, la consommation de poisson a décliné autour du lac en raison, notamment, de la priorité accordée à l'exportation des filets de perche (Jansen et al., 1999). Pire, constate-t-il, « les études montrent également que dans de nombreuses communautés autour du lac, près de la moitié des enfants souffrent de malnutrition ». Or, nous avons vu qu'il n'existe aucune malnutrition dans la région du lac. Un autre argument fort de E.G. Jansen concerne le déséquilibre constaté sur le marché du travail, entre hommes et femmes. Auparavant, les femmes servaient d'intermédiaires entre les pêcheurs et le marché. Désormais, le quasi-captage des captures par l'industrie de filetage les prive de leur activité et inverse ainsi le marché au profit des pêcheurs qui traitent directement avec les usines. Ce constat est peut-être vrai, mais n'oublions pas cependant que la majorité des emplois fournis par les usines d'exportation est dévolue aux femmes. Est-ce une reconversion ou une véritable perte d'activité? Jansen ne dit rien à ce propos. Mais d'autres ont leur opinion et pensent que le secteur informel, préconisé par E.G. Jansen, n'est bien souvent qu'un débouché forcé et involontaire (Trani, 2006). Enfin, l'économiste pronostiquait, à très brève échéance, un tarissement de cette industrie d'exportation. À l'heure actuelle, sa prévision est non seulement erronée, mais il semble bien que nous n'observions pas de véritable cycle de récession. En effet, conscients d'une possible surexploitation, les États ont pris les mesures nécessaires pour que le Lates constitue une ressource durable. En conclusion, cet «avocat des petits boulots » (Garçon, 2006b) a émis plusieurs hypothèses qui se sont toutes révélées peu fiables. 


\section{Encadré 4. Mauvaise foi pour... une bonne cause}

Certains médias, ayant admis la supercherie, ont néanmoins maintenu leur appui au film pour la raison que, parfois, la fin justifie les moyens. Écoutons ce qu'en pense Philippe Val : « La mauvaise foi n'est jamais au service d'une bonne cause mais au service de celui qui est de mauvaise foi pour obtenir une victoire personnelle en utilisant une cause. [...] La misère en Afrique est une réalité. Les remèdes qu'il faut trouver pour en faire disparaître les causes sont complexes. Personne n'a la solution miracle, mais on sait au moins une chose : le mensonge n'en est pas une ${ }^{15}$. »P. Val va plus loin et cite Prévert : «Lorsque H. Sauper se défend: "Qu'est-ce que ça peut faire que je sois de mauvaise foi puisque c'est pour la bonne cause?", Prévert répond: "Et qu'est-ce que ça peut faire que je sois pour la mauvaise cause puisque c'est de bonne foi ?" » Bien entendu, chacun est libre de distribuer bons ou mauvais points selon sa conviction. Mais nous faisons nôtre la réponse du poète, pas l'argument du «documentariste».

\section{Clap de fin}

Rappelons les principales idées véhiculées par le film : 1. Les populations locales sont privées de poissons à cause de l'exportation de la perche; 2 . L'industrie de la perche a contribué à faire disparaître les emplois; 3. L'exportation de la perche contribue à la famine; 4 . Les bénéfices rapportés par la perche ne sont pas restitués à la population.

Le dernier message, certainement pas le moins anodin, que veut faire passer $\mathrm{H}$. Sauper concerne le trafic d'armes. Nous n'avons aucune compétence pour nous prononcer sur ce trafic qui revient comme un refrain tout au long du film, mais F. Garçon (2006b) s'insurge contre cette thèse, car aucune image n'est fournie. S'il en avait eu, H. Sauper ne se serait probablement pas privé de les montrer. On voit donc que l'ensemble des idées véhiculées durant ce long métrage n'est que suggéré. La globalité relève de la «méthode Couet » et, en y regardant d'un peu plus près, on note que chaque opinion présentée n'a aucun fondement véritable et n'est distribuée que pour servir la cause militante défendue par l'auteur.

Cela peut aller très loin dans le voyeurisme. Que penser de ces enfants qui se battent pour une gamelle de riz ? Cette scène est d'évidence jouée, "scénarisée ", et personne n'est dupe. H. Sauper aurait-il donc travesti la réalité à propos de la famine? Au-delà, aurait-il agi de la sorte pour le trafic d'armes ou pour d'autres sujets? Ce qui est certain, c'est que la vérité scientifique est, elle aussi, fortement sujette à caution. Le film Le Cauchemar de Darwin suggère donc, sans jamais rien démontrer et en s'écartant de l'analyse scientifique pour laisser place à l'affectif. Non, l'introduction de la perche du Nil dans le lac Victoria n'est pas à l'origine d'une paupérisation dramatique des populations riveraines.
Le film a pourtant, en partie, atteint son but. Jamais à court de combat, mettant en avant leur courageuse prise de position bien-pensante, quelques militants ont également trouvé là l'occasion de montrer leur solidarité. Mais solidarité envers qui ? C'est là que le bât blesse! Il s'est, en définitive, trouvé peu d'Africains pour leur emboîter le pas. Après tout, pour aider les populations locales, faut-il prôner le boycott d'un produit qui les fait vivre?... C'est la bataille de ces grands donneurs de leçons : décider pour les pauvres. C'est montrer bien du mépris en laissant croire que les Africains ne peuvent décider et donc raisonner pour eux-mêmes.

En conclusion, les ravages provoqués par ce genre de film découlent de deux raisons essentielles. La première est que personne ne semble s'être posé la question de la vérité de ce qui est affirmé. Il est acquis que le «documentariste » nous dit la vérité, il est acquis que nous visionnons un film-vérité (Encadré 4). La seconde, corollaire de la précédente, est que, dans un premier temps, les médias n'effectuent aucune vérification et relaient le message d'autant plus facilement que les images qui nous sont fournies éveillent la morbidité.

Bref, un bilan global qui n'est sans doute pas celui dont rêvait H. Sauper, du moins dans son épilogue. Mais reconnaissons-lui, néanmoins, d'avoir, par sa prise de position, permis de faire émerger le débat. Nous lui en savons gré, mais nous ne sommes pas certains que, à l'heure actuelle, tous soient dans cet état d'esprit.

\section{Références}

Almarcha, G., 2005. Le Cauchemar de Darwin, un film qui réalise $L a$ Fin du développement, Natures Sciences Sociétés, 13, 4, 437-439.

Caraco, N.F., 1995. Influence of human populations on phosphorus tranfers to aquatic systems: a regional scale study using large rivers, in Tiessen, H. (Ed.), Phosphorus in Global Environment, New York, Wiley, 235-244.

Courade, G. (Ed.), 2006. L'Afrique des idées reçues, Paris, Belin.

FAO, 2006. FishStat Plus (Version 2.3). Universal software for fisheries statistical time series (http://www.fao.org/fi/ website/FIRetrieveAction.do?dom=topic \& fid=2017).

Garçon, F., 2006a. Le Cauchemar de Darwin : allégorie ou mystification, Les Temps modernes, 635-636, 353-379.

Garçon, F., 2006b. Enquête sur Le Cauchemar de Darwin, Paris, Flammarion.

Goldschmidt, T., 2003. Le Vivier de Darwin : un drame dans le lac Victoria, Paris, Le Seuil.

Goudswaard, K. (P.C.), Witte, F., 1997. The catfish fauna of Lake Victoria after the Nile perch upsurge, Environmental Biology of Fishes, 49, 1, 21-43.

Goudswaard, P.C., Witte, F., Katunzi, E.F.B., 2002. The tilapiine fish stock of Lake Victoria before and after the Nile perch upsurge, Journal of Fish Biology, 60, 4, 838-858.

Graham, M., 1929. The Victoria Nyanza and its Fisheries: A Report on the Fishing Survey of Lake Victoria, 1927-1928, and Appendices, Crown Agents for the Colonies, London.

\footnotetext{
15 Philippe Val, dans Charivari, France Inter, 13 mars 2006 (cité
} par F. Garçon, 2006b). 
Jansen, E.G., Abila, R.O., Owino, J.P., 1999. Constraints and Opportunities for "Community Participation" in the Management of the Lake Victoria Fisheries. Socio-economics of the Lake Victoria Fisheries, IUCN Eastern Africa Programme, Rep. No. 6 (http://www.iucn.org/places/earo/pubs/wetlands/ constraints.pdf).

Kaufmann, L., 1992. Catastrophic change in species-rich freshwater ecosystems: the lessons of Lake Victoria, BioScience, 42, 11, 846-858.

Lévêque, C., Paugy, D., 2006. Le paradoxe de Darwin, La Recherche, 402, 48-51.

Matsuishi, T., Muhoozi, L., Mkumbo, O., Budeba, Y., Njiru, M., Asila, A., Othina, A., Cowx, I.G., 2006. Are the exploitation pressures on the Nile perch fisheries resources of Lake Victoria a cause for concern?, Fisheries Management and Ecology, 13, 1, 53-71.

Meyer, A., 2005. Evolutionary Biology: Cichlid species flocks of the past and present, Heredity, 95, 6, 419-420.

Modesta, M., 2005. The Nile perch processing industry in Tanzania: trends, issues and development, in Seventh FAO Expert Consultation on Fish Technology in Africa, Saly-Mbour, Republic of Senegal, 10-13 December 2001. Report No 712, FIIU/R712 (Bi), FAO, Rome, 149-154 (ftp://ftp.fao.org/docrep/ fao/008/y9155b/y9155b00.pdf).

Nelson, J.S., 2006 [4th ed.]. Fishes of the World, Hoboken (N.J.), John Wiley.

Nishida, M., 1991. Lake Tanganyika as an evolutionary reservoir of old lineages of Esat African cichlid fishes: inferences from allozyme data, Experentia, 47, 277-290.

Ogutu-Ohwayo, R., 1990. The decline of the native fishes of lakes Victoria and Kyoga (East Africa) and the impact of introduced species, especially the Nile perch, Lates niloticus, and the Nile tilapia, Oreochromis niloticus, Environmental Biology of Fishes, 27, 2, 81-96.

Pitcher, T.J., Hart P.J.B. (Eds), 1995. The Impact of Species Changes in African Lakes, London, New York, Chapmann \& Hall.
Pringle, R.M., 2005. The origins of the Nile perch in Lake Victoria, BioScience, 55, 9, 780-787.

Salzburger, W., Meyer, A., 2004. The species flocks of East African cichlid fishes: Recent advances in molecular phylogenetics and populations genetics, Naturwissenschaften, 91, 277-290.

Salzburger, W., Mack, T., Verheyen, E., Meyer, A., 2005. Out of Tanganyika: Genesis explosive speciation, keyinnovations and phylogeography of the haplochromine cichlid fishes, BMC Evolutionary Biology, 5, 17 (disponible sur le site Internet : http://www.biomedcentral.com/content/ pdf/1471-2148-5-17.pdf).

Scheren, P.A.G.M., Zanting, H.A., Lemmens, A.M.C., 2000. Estimation of water pollution sources in Lake Victoria, East Africa: Application and elaboration of the rapid assessment methodology, Journal of Environmental Management, 58, 4, 235-248.

Stiassny, M.L.J, Meyer, A., 1999. Cichlids of the rift Lakes, Scientific American, 280, 64-69.

Tate, E., Sutcliffe, J., Conway, D., Farquharson, F., 2004. Water balance of Lake Victoria: update to 2000 and climate change modelling to 2100, Hydrological Sciences Journal, 49, 4, 563-574.

Trani, J.-F., 2006. L'économie informelle est la voie pour un développement à l'africaine, in Courade, G. (Ed.), L'Afrique des idées reçues, Paris, Belin, 370-375.

Verschuren, D., Johnson, T.C., Kling, H.J., Edgington, D.N., Leavitt, P.R., Brown, E.T., Talbot, M.R., Hecky, R.E., 2002. History and timing of human impact on Lake Victoria, East Africa, Proceeding of the Royal Society B, 269, 1488, 289-294.

Witte, F., Goldschmidt, T., Wanink, J., van Oijen, M., Goudswaard, K., Witte-Maas, E., Bouton, N., 1992. The destruction of an endemic species flock: quantitative data on the decline of the haplochromine cichlids of Lake Victoria, Environmental Biology of Fishes, 34, 1, 1-28.

Worthington, E.B., 1932. A Report on the Fisheries of Uganda: Investigated by the Cambridge Expedition to the East African Lakes, 1930-31. Crown Agents for the Colonies, London. 